\title{
System Planning of Route Diagram for China Railway Passengers Based on Network and Ergonomics
}

\author{
Junmin YI \\ School of Management, Xiamen University of Technology, Xiamen 361024, China \\ E-mail: yijunmin@xmut.edu.cn
}

\begin{abstract}
Focus on the China Railway network and its planar route diagram for passengers, this study further extends network analysis to the representation of the railway network. After detail system analysis, backbone framework for the China Railway and its high-speed railway network is identified. With a straight-line principle, planning horizon till the year of 2020, and ergonomic pictogram measures, a brand new route diagram for China Railway passengers is created. This diagram will convey a new image and modern information of China Railway to meet the demand of passengers and the requirement of ever-expanding network.
\end{abstract}

Keywords network; system; railway; ergonomics; route diagram

\section{Introduction}

Since the new century, the construction of railway has accelerated by the Chinese Government to improve the national infrastructure and to meet the ever-growing demand of passengers and cargos. In this boom era, the China Railway has expanded an annual mileage of over 2000 kilometers in the 2000-2010 period, while 8358 kilometers are the emerging high-speed railway. In 2013, with the transform of Ministry of Railway to the new China Railway Corporation, The Corporation is still planning to increase its mileage over 3000 kilometer annually in recent years. The high-speed railway network ( $\mathrm{CRH}$, China Railway High-speed), especially the Beijing-Shanghai, Beijing-Guangzhou High-speed Railways have drawn attentions from the whole world. Although the ambitious high-speed railway network has come under sustained criticism in recent years for delays, safety concerns, rigid system and ticket price, the railway is still and will be a major way for passengers, the administrative, industries and academics should cooperate to improve it. In the process for better service, speed, safety and robustness, there are still a long way for the new China Railway Corporation to strive for. Today, the China Railway has a network with annual passenger throughput of 2 billion persons and a total service mileage over 100 thousand kilometers, on which runs 2369 pairs of passenger train with more than 4 million seats daily.

For transport services, it is common to find diagram maps, which are two-dimensional geometric symbolic drawings using simple lines rather than realistic geographic details to illustrate a transportation structure in a certain area, such as metros ${ }^{[1]}$, buses, airlines or railways. A

Received November 27, 2013, accepted January 6, 2014

Supported by NSFC (Grant No. 71371162). 
route diagram map serves all walks of passengers and customers with different literacy and vast backgrounds, and shows them the origins, destinations and transfer nodes quickly from dense networks. In China specially, there are diagram maps for railway passenger's lines by the Ministry of Railway ${ }^{[2]}$. But those diagram maps were not systematic planned and their design is lack of system consideration. Their main structure is based on railway network decades ago, and because of their rigidness of lines, shortage of aesthetics, lack of the convenience, the old diagram is hard to be recognized by normal passengers ${ }^{[3]}$. Subsequently, our purpose is to systematically and ergonomically design the diagram map for passengers' lines of China Railway to meet the huge demand of passengers and the requirement of ever-expanding network.

Nevertheless, the research of complex network shed light on the difficulty. Recent years with the emerging study of complex networks ${ }^{[4]}$, some scholars begin the research of transportation networks which exhibit some characteristics that are not necessarily shared by other types of networks ${ }^{[5]}$, such as small sizes (compared to the large networks such as the Internet), relatively stable spatial $\mathrm{C}$ temporal structures, and weighted links ${ }^{[6]}$. The very complex topological structures of railway networks have attracted the attention of researchers in many different contexts. For example the fractal nature of the structure of railway networks was studied by Benguigui and Daoud ${ }^{[7]}$, Stanley ${ }^{[8]}$. The studies of the Boston subway ${ }^{[1]}$, Indian Railway network ${ }^{[9]}$, Boston and Vienna subway ${ }^{[10]}$, Shanghai subway network ${ }^{[11]}$, Chinese Railway network $^{[12]}$, Souel Subway ${ }^{[13]}$ and metro systems in the world ${ }^{[14-15]}$ have revealed an important commonality: most transportation networks are endowed with small-world properties.

However, these scholars just focused on the study of network property itself, they seldom further extend to the representation of the railway network. Our study aims on the diagram map of the China Railway network for billions of passengers, to gather existing and propose new applications by network analysis. The rest of the paper is organized as follows: Section 2 discusses recent developments in the analysis of railway networks and a backbone erection. Section 3 describes the development of the final diagram map and its characteristics, while Section 4 presents ergonomic measures and Section 5 with concluding remarks.

\section{Railway network analysis}

The railway networks were usually visualized by nodes representing stations or spots and by links between them representing their connections. Based on the meanings of links, two kinds of network can be constructed, firstly geographic network with links of railway segment which connects two stations; secondly the traffic network with links of train access of two adjacent stations. Studies of geographic network show that it looks more like a tree with several trunks and many branches, and traffic network shows topology properties such as small-world behavior and scale-free structure ${ }^{[16]}$. However, our study is focused on the former one.

\subsection{Study of China railway network}

The China Railway network has a data set of nearly 30000 which is larger than most transportation networks previously studied. Therefore, it is reasonable to attract more study on this network. Firstly, Jin, Wang and Sun ${ }^{[17]}$ first studied the spatial impact on China's railway traffic network and further conducted the analysis of China Railway network expansion and its

spatial accessibility ${ }^{[12]}$. Zhao et al ${ }^{[18]}$ studied both the geographic and traffic networks of China 
Railway, and showed that the former one is a tree-network with approximately zero average clustering coefficient (indicates the extent to which the nodes in the neighborhood of a certain node are connected), while the later is a small world network with scale-free property, which has comparatively large average clustering coefficient (0.83) and small average path length (3.27, which means after an average of 3.27 times of transfer, any of two stations in the China Railways network can be reached.), and the degrees of most nodes of the traffic one follow a scale-free degree distribution. In the empirical analysis ${ }^{[6]}$, the statistical properties of the China Railway Network (CRN) consisting of 3915 nodes (train stations) and 22259 edges (railway segments). With newer data, they have verified that CRN's small-world network characteristics with similar results as Zhao's. Empirical analysis by Wang et al ${ }^{[16]}$ found that the distribution of the path length of every CRN train run decays as power law, the distribution of the railroad length per degree follows the shifted power law and the distribution of stations is close to the optimal. Wu et al ${ }^{[19]}$ investigate the accessibility pattern of China Railway passenger transport network by choosing the spatial distance, temporal distance, connectivity and selectivity as the indicators. Wang et al ${ }^{[20]}$ analyzes the expansion of China's railway network, the evolution of its spatial accessibility, and the impacts on economic growth and urban systems over a time span of 19062000. Tang et al ${ }^{[21]}$ investigated the degree distribution and degree correlation coefficient of 3182 CRN stations (nodes), and their numerical simulations showed the passenger volume of a station is proportional to the weight of corresponding node, while nonlinear to the degree of the node.

It is noted that most China Railways are hybrid lines both for passenger and cargo trains, the new High speed railways are usually passenger dedicated railways parallel to old lines to release the old hybrid one mostly for cargo.

However, with the rapid development of China Railway network, especially recent years in big cities with multiple railway terminals, to separated these station in one city to several nodes in CRN is not rational from the passenger's perspective. Connectivity between cities should be first concept of railway network, hence in our discussion, a large city-level hub node is used to include all railway stations, such as Beijing hub to include all the four passenger stations of Beijing, Beijing West, Beijing South and Beijing North, Shanghai hub with Shanghai Station, Shanghai South, Shanghai Hongqiao but small stations in suburban such as Jiading and Songjiang are ignored. Then, to forming of the network diagram map, first is to identify its backbone network.

From previous study, it is known that railway geographic network in general shows treenetwork property but still with some circuits, these redundancies in connectivity generate better options and service level in case of disaster breakout one or more links. Nevertheless, by importance and node degree, those nodes in circuits erect our backbone network.

\subsection{Backbone of CRN network}

Apparently, large cities in China have more possibility to be a backbone node. The spatial pattern of railway network expansion in China investigated by Jin and Wang ${ }^{[11]}$ gives us a primitive image of the forming of CRN backbone. The Chinese Ministry of Railway listed six passenger hubs: Beijing, Shanghai, Guangzhou, Wuhan, Chengdu and Xi'an. By network analysis, Zhao et al ${ }^{[18]}$, Wu et al ${ }^{[19]}$ have identified and compared eight railway hubs in 
China, which are Beijing, Shanghai, Guangzhou, Zhengzhou, Wuhan, Shenyang, Chengdu and Lanzhou. Whichever top hub cities are, they are all seats of Railway Administration and at least provincial-capital-level cities. Thereafter, we choose the key cities of those municipalities, provincial capital cities and the five sub-provincial coastal cities Dalian, Qingdao, Ningbo, Xiamen and Shenzhen as our backbone vertices base, in which only Macau has no railway connection yet and Taipei is not connected to mainland network.

On the other way, the edges for backbone should be considered; several corridors come to the option base. The four vertical and four horizontal $(4 \mathrm{~V} 4 \mathrm{H})$ corridors proposed by MOR is a natural choice here. Hence, our backbone consists of the $4 \mathrm{~V} 4 \mathrm{H}$ and their main stations, as shown in Figure 1. This is a graph with 34 vertices and 41 edges.

\section{Developing the route diagram}

After network analysis, our network should go to planar representation - route diagram map - it is not a real map with exact geographic nodes and line curve but a simple clear vector drawing to represent the railway network. The finished diagram (Figure 2) includes 372 cities/stations in a $152 * 114$ lattice, with space for more stations in the future. The diagram reveals several interesting results.

- In general, there are more horizontal lines than vertical ones, but the North to South vertical backbone lines are more strong and in evidence.

- Slope lines lie more in the West and the South, with a reflection of less orthogonal and straight lines in these mountainous regions.

- There is a distinct difference between the South and the North by the Gansu-Jiangsu Line. There are more curves in the South, while more straight lines in the North, and especially in the vast Northwest China, the sparse railway lines can be drawn just straightforward.

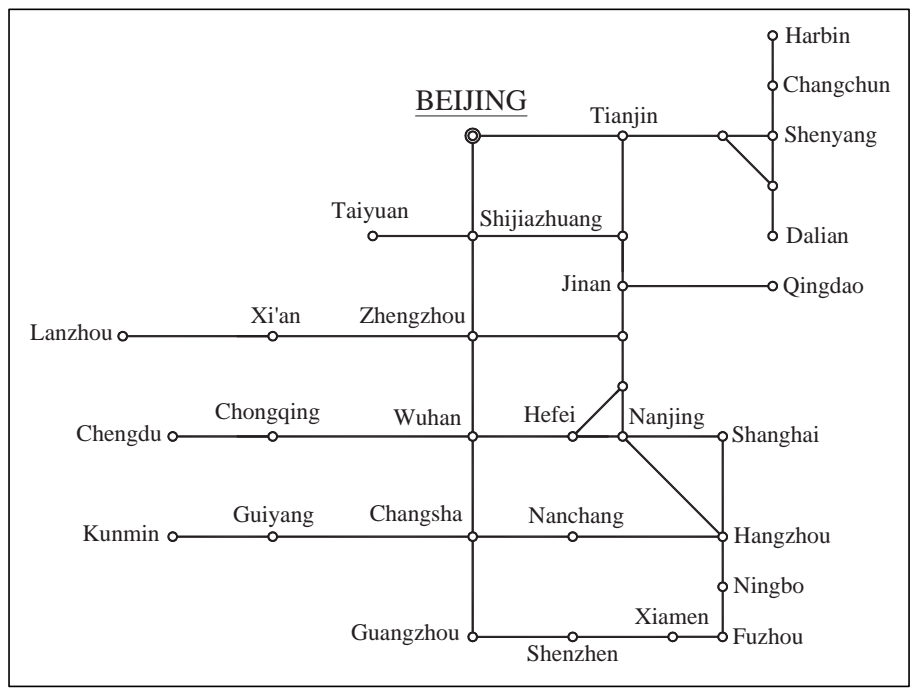

Figure 1 Backbone of CRN network 


\subsection{Straightening of main lines}

The backbone network is formed with straight segments as many as possible, the $4 \mathrm{~V} 4 \mathrm{H}$ are set in 21 vertical and 20 horizontal segments. For example, in the $1318 \mathrm{~km}$ long Beijing-Shanghai High-speed Railway, there are 24 stations located on 17 cities widespread in seven provinces. In our map, fifteen important cities are listed on a z-type line. Also, it straightens most of the dual eight main Vertical and Horizontal railway lines $(8 \mathrm{~V} 8 \mathrm{H})$ by the finished the tenth FiveYear Plan such as the Beijing-Kowloon, Lanzhou-Xinjiang, Datong-Zhanjiang, Baotou-Liuzhou (Baotou-Guiyang section), and Lanzhou-Kunming (Chengdu-Guangtong section) Lines, and many other existing railways in North China. Even the Datong-Qinghuangdao Line mainly for cargo transport is represented in straight line on our diagram map. In all, the diagram map conveys a backbone straightforward high-speed scheme, which China Railway is undergoing.

\subsection{Based on the long term planning}

As mentioned in Section 1, the railway construction is booming in China these years with an annual 3\% 5\% leap in line length and network expansion, an updated route diagram should catering to the future. Hence we hold a planning horizon as the Revised Mid and Long term Railway Planning with a horizon till the year of 2020. It focuses on the network connection and grid space to meet the requirement of present framework and future development.

\subsection{Density and degree}

By density, the highest lies in Northeast China and The Yangtze River delta. There are more lines by planning and under construction in the regions of East China (South) and Southwest China, which reflects the potential demand of railway service.

For the nodes in the diagram map, their vertex degree can be applied to explain the connecting relationship. Beijing as the top railway hub has a vertex degree of 12, which means there are twelve railway lines started from Beijing. Next, Nanjing, Wuhan and Xi'an both have a degree of 10. Shanghai, Guangzhou, Zhengzhou, Chongqing and Chengdu have a degree of 8 or 9. These hubs are also large cities in China with great population and passengers.

\section{Ergonomic measures on $\mathrm{CRN}$ route diagram}

A passenger-favorite route diagram should consider the human factors. Here, the route diagram of Paris metro, Boston subway ${ }^{[1]}$ etc can serve as good examples. In such diagram, there are explicit rules on pictograms, such as stations, line type and curve, colors and fonts, which ergonomic principles applied ${ }^{[22]}$.

\subsection{The nodes by type}

The nodes are on the top priority for passengers to find the origin and destination from the diagram quickly. In our design, five classes of node are classified:

- Class 1. Beijing is represented by a pentagram star in a large circle for the normal meaning of state capital and top railway hub.

- Class 2. Cities where railway administrative bureau seated are represented by a dual large circle, these 18 cites are both provincial capital now. 
- Class 3. Other provincial capital cities and five large sub-provincial level cities are represented by a large circle.

- Class 4. Cities in the intersection of several lines, especially with both normal line and $\mathrm{CRH}$ line are represented by a long circle.

- Class 5. Other stations are represented by a small circle.

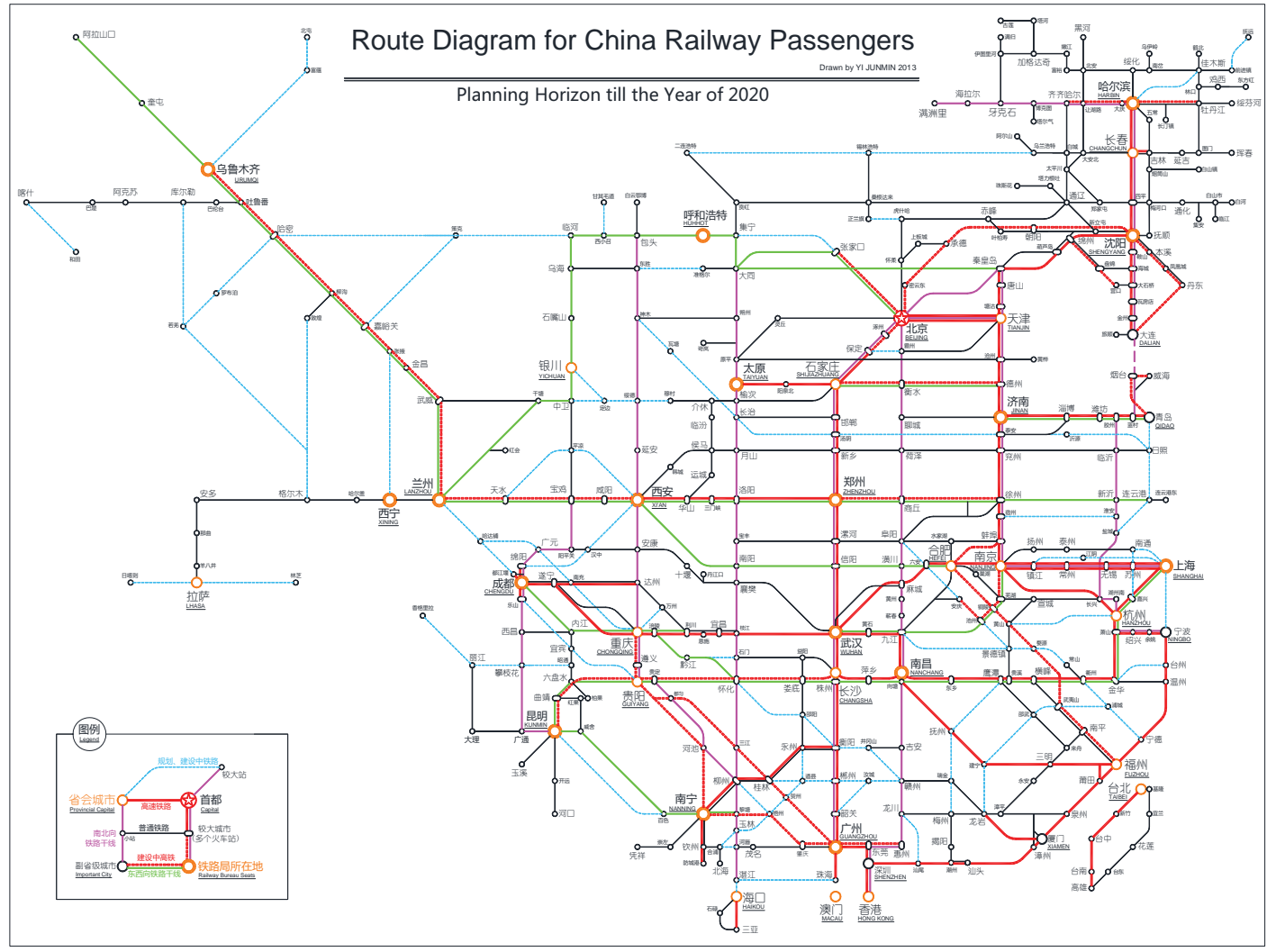

Figure 2 Finished route diagram

\subsection{Font for nodes}

The font of stations and cities are necessary and meaningful to read the diagram. In our design, the font is always in black san serif for clear reading, and there are four types with two fonts.

- Type 1 is for Class 1 and 2 cities by Microsoft Yahei font with font height of 5;

- Type 2 for Class 3 cities by Youyuan font with font height of 5 ;

- Type 3 for Class 4 by Youyuan font with font height of 3.5;

- Type 4 for Class 5 stations by Microsoft Yahei font with font height of 2.5.

The ratio of font height is 1:0.7:0.5 close to the golden section, which has a clear differentiation. 


\subsection{Line types, coloring and curves}

According to the principles of ergonomics and visual communication, four types of line and color are used to classifying different types of railway.

- A red line with line width of 1 is for the latest CRH High-speed railway service, while a dashed one for such railway under construction, an example is the new Wuhan-Guangzhou $\mathrm{CRH}$ line paralleling to the old ones (one of the $8 \mathrm{~V}$ in pink with line width of 0.6 , while $8 \mathrm{H}$ in green).

- A wathet blue line with line width of 0.3 is for planned lines while black one for existing line with rated speed less than $200 \mathrm{~km} /$ hour.

The colors are chosen by railway tradition and general color meanings. The ratio of line width is 1:0.6:0.3, which has a clear variation.

While links are straightened and laid orthogonally as many as possible, non vertical or horizontal lines are laid only in 45 degree orthogonal with curved turning for streamline effect.

\section{Concluding remarks}

To summarize, our study focus on the China Railway Network (CRN) and its representation in route diagram; after investigation of the network properties of CRN, we initially proposed the backbone of CRN, while nodes are cities rather than stations, the links are defined as the railway segments connecting two pairs of nodes. With a concept of straightening links and catering for future, a brand new route diagram for China Railway passengers is created by applying selected pictograms and ergonomic measures. This new route diagram is expected to meet the demand of billions of passengers and the requirement of ever-expanding CRN network.

\section{Acknowledgements}

The author is grateful to the HKSTS for poster and discussion of the primary paper, also to the editors and the anonymous referees for their constructive comments and valuable suggestions which have helped me very much to improve the paper.

\section{References}

[1] Latora V, Marchiori M. Is the Boston subway a small-world network? Journal: Physica A — Statistical Mechanics and its Applications, 2002, 314(1): 109-113.

[2] MOR Transportation Bureau. The diagram map for passengers' line in China Railways. China Railway Press, Beijing, 2009.

[3] Yi J M, Yuan H B, Zhang Q. Drawing of railway passenger line scheme towards passenger and ergonomics. Chinese Railways, 2010(9): 4-7.

[4] Strogatz S H. Exploring complex networks. Nature, 2001, 410: 268-276.

[5] Lin J Y, Ban Y F. Complex network topology of transportation systems. Transport Reviews, 2013, 33(6): 658-685.

[6] Liu C M, Li J W. Small-world and the growing properties of the China Railway network. Frontiers of Physics in China, 2007, 2(3): 364-367.

[7] Benguigui L, Daoud M. Is the suburban railway system a fractal? Geographical Analysis, 1991, 23(4): 362-368.

[8] Stanley H E. Fractal landscapes in physics and biology. Physica A: Statistical Mechanics and its Applications, 1992, 186(1): 1-32. 
[9] Sen P, Dasgupta S, Chatterjee A, et al. Small-world properties of the Indian railway network. Physical Review E, 2003, 67, 036106.

[10] Seaton K A, Hackett L M. Stations, trains and small-world networks. Physica A: Statistical Mechanics and its Applications, 2004, 339(3): 635-644.

[11] Zhang J H, Xu X M, Hong L, et al. Networked analysis of the Shanghai subway network, in China. Physica A: Statistical Mechanics and its Applications, 2011, 390(23): 4562-4570.

[12] Jin F J, Wang J E. Railway network expansion and spatial accessibility analysis in China: 1906-2000. Acta Geographica Sinica, 2004, 59(2): 293-302.

[13] Lee K, Jung W S, Park J S, et al. Statistical analysis of the Metropolitan Seoul Subway System: Network structure and passenger flows. Physica A: Statistical Mechanics and its Applications, 2008, 387(24): 62316234 .

[14] Derrible S, Kennedy C. The complexity and robustness of metro networks. Physica A: Statistical Mechanics and its Applications, 2010, 389(17): 3678-3691.

[15] Roth C, Kang S M, Batty M, et al. A long-time limit for world subway networks. Journal of the Royal Society Interface, 2012, 75(9): 2540-2550.

[16] Wang R, Tan J X, Wang X, et al. Geographic coarse graining analysis of the railway network of China. Physica A: Statistical Mechanics and its Applications, 2008, 387(22): 5639-5646.

[17] Jin F J, Wang J E, Sun W. Spatial impacts of China's train network behind its upgrading: 1995-2002. Journal of the China Railway Society, 2003, 25(6): 1-7.

[18] Zhao W, He H S, Lin Z C, et al. The study of properties of China railway passenger transport network. Acta Physica Sinica, 2006, 55(8): 3906-3911.

[19] Wu W, Cao Y H, Liang S B, et al. The accessibility pattern of railway passenger transport network in China. Geographical Research, 2009, 28(5): 1389-1400.

[20] Wang J E, Jin F J, Mo H H, et al. Spatiotemporal evolution of China's railway network in the 20th century: An accessibility approach. Transportation Research Part A: Policy and Practice, 2009, 43(8): 765-778.

[21] Tang F R, Yang X Q, Tang G, et al. Study of the topology of Chinese rail network and its passenger flow. Journal of China University of Mining \& Technology, 2010, 39(6): 935-940.

[22] Sanders M S, McCormick E J. Human factors in engineering and design. 7th ed. McGraw-Hill, New York, 2002. 